\title{
On The Randomized Schmitter Problem
}

\author{
Hansjörg Albrecher ${ }^{1}$ (D) . José Carlos Araujo-Acuna ${ }^{2}$ D
}

Received: 22 December 2020 / Revised: 3 October 2021 / Accepted: 17 October 2021 /

Published online: 8 November 2021

(c) The Author(s) 2021

\begin{abstract}
We revisit the classical Schmitter problem in ruin theory and consider it for randomly chosen initial surplus level $U$. We show that the computational simplification that is obtained for exponentially distributed $U$ allows to connect the problem to $m$-convex ordering, from which simple and sharp analytical bounds for the ruin probability are obtained, both for the original (but randomized) problem and for extensions involving higher moments. In addition, we show that the solution to the classical problem with deterministic initial surplus level can conveniently be approximated via $\operatorname{Erlang}(k)$-distributed $U$ for sufficiently large $k$, utilizing the computational advantages of the advocated randomization approach.
\end{abstract}

Keywords Schmitter problem · Ruin theory $\cdot$ Ruin probability $\cdot$ Laplace transform • Stochastic ordering $\cdot$ Erlangization

\section{Introduction}

At the ASTIN Colloquium 1990 in Montreux, the Swiss actuary Hans Schmitter presented an algorithm for the exact evaluation of the ruin probability $\psi(u)$ of a CramérLundberg surplus process for an insurance portfolio with initial surplus $u$, for the case when the claim amount distribution is discrete on a finite range (Schmitter (1990)). Also inspired by Bowers (1969), he then posed the following question: If the individual claims are known to have mean $\mu$ and variance $\sigma^{2}$, which claim size distributions minimize or maximize the ruin probability for a given $u$, respectively? I.e., the problems are

Hansjörg Albrecher

hansjoerg.albrecher@unil.ch

José Carlos Araujo-Acuna

jose.araujo@stat.unibe.ch

1 Department of Actuarial Science, Faculty of Business and Economics, University of Lausanne, Switzerland and Swiss Finance Institute, Lausanne, Switzerland

2 Department of Mathematics and Statistics, Institute of Mathematical Statistics and Actuarial Science, University of Bern, Bern, Switzerland 


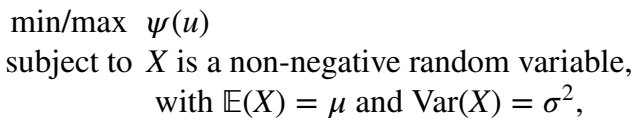

where $X$ is the random variable describing the individual claim sizes. This problem was then further discussed by Brockett et al. (1991) and taken up in Kaas (1991), where it was also extended to the related problem of finding extremal values of stop-loss premiums for compound Poisson distributions with similar moment restrictions. Much later, De Vylder et al. (1997b); De Vylder and Marceau (1996) provided a numerical solution to the Schmitter problem based on a renewal equation that approximates the classical ruin model using a discrete time grid and partially solved the original problem in De Vylder et al. (1997a).

While on the basis of these contributions the problem can be considered as quite well understood, it was never solved in full generality. Correspondingly, despite the considerable time that has passed since then and the gradual shift of criteria for solvency considerations in insurance practice in the meantime, we would like to add an additional layer of complexity and understanding of the Schmitter problem in this paper by taking the perspective of a randomized initial surplus level.

Randomization as a principle has proven to be a very useful tool in risk theory leading to simpler expressions (see e.g. Albrecher et al. (2013), Ivanovs (2013)) or even unexpected identities (Albrecher and Ivanovs (2017)), but particularly also to considerable computational advantages (cf. Carr (1998), Asmussen et al. (2002), Albrecher and Goffard (2021)). The idea for the latter computational approach is to replace a deterministic quantity by a random variable with matching expected value, often with the advantage of smoothing the corresponding computational problem, leading to simpler and amenable expressions. In a final step, if possible the variance of that random variable is reduced considerably such that the resulting value can be an excellent approximation of the original computationally complex problem ("Erlangization").

In our setting, we replace the deterministic initial surplus level $u$ by an exponentially distributed random variable $U$ with mean $u$. The expected value of the resulting ruin probability can then be expressed in terms of the (simpler) Laplace transform of the classical ruin probability under the Cramér-Lundberg model. At this level, analytical lower and upper bounds for the ruin probability in the Schmitter problem can then be established utilizing the strong results in the theory of $m$-convex orders obtained by Denuit et al. (1999, 1998) (see also Lefèvre et al. (2020) for a recent application of this ordering concept). In addition, the generality of the latter results in fact allows to give sharp upper and lower bounds for the ruin probability when more than two moments of the underlying claim size distribution are specified, which can be seen as an extension of the Schmitter problem that naturally narrows the gap between the upper and lower bound. For a comprehensive survey of stochastic orderings we refer to the monographs by Kaas et al. (1994), Shaked and Shanthikumar (1994, 2007) and Müller and Stoyan (2002). More recent treatments in a specifically actuarial context include Kaas et al. [Ch.7] (2008) and Asmussen and Steffensen [Ch.8] (2020).

Eventually, we are also interested in using these explicit expressions of the randomized model to approximate the classical situation of deterministic initial surplus level $u$. Developing the results further towards $\operatorname{Erlang}(k)$ distributed initial surplus, for increasing $k$ (maintaining the expected value at $u$ ) this provides increasingly accurate approximations for the classical deterministic case, expressed through the explicit formulas of the randomized model. 
The remaining paper is structured as follows. First, Section 2 recapitulates the model setting and summarizes relevant results from the existing literature. In Section 3 we then analyze the problem for an exponentially distributed initial surplus level $U$. We obtain an expression for the corresponding (expected) ruin probability in terms of the Laplace transform of the classical ruin probability in the Cramér-Lundberg model, and provide sharp lower and upper bounds for it when the claim size is bounded. We also provide corresponding bounds in the case of more than two pre-specified moments of the claim size distribution. Moreover, we illustrate the resulting interval for particular numerical parameters and place various concrete (truncated) claim size distributions within these bounds. In Section 4, we expand the randomization idea towards Erlang $(k)$-distributed initial surplus, and in the spirit of Asmussen et al. (2002) we approximate the ruin probability with deterministic surplus via Erlangization and Richardson extrapolation. We give numerical illustrations which show that the known and somewhat curious kinks in the graphs of the known optimal solutions of the classical Schmitter problem can be smoothly approximated with this randomization approach. In some cases, a small value of $k$ is already sufficient for a good approximation, in others the value of $k$ has to be quite considerable. Section 5 concludes.

\section{Preliminaries and Previous Results}

Consider the classical Cramér-Lundberg model with surplus process

$$
C(t)=u+c t-S(t),
$$

at time $t \geq 0$, where $u$ is the initial surplus level. Here, $S(t)=X_{1}+\cdots+X_{N(t)}$ denotes the aggregate claims up to time $t$, where the number of claims $\{N(t) ; t \geq 0\}$ up to time $t$ refers to a homogeneous Poisson process with rate $\lambda>0$ and the claim sizes $X_{i}, i=1,2, \ldots$, are independent and identically distributed random variables with distribution function $F_{X}$ and expected value $\mathbb{E}\left(X_{1}\right)=\mu$, independent of $\{N(t) ; t \geq 0\}$. We assume that all moments of $X_{1}$ exist. The premium income per unit of time is $c=(1+\theta) \lambda \mu$, where $\theta>0$ is the safety loading. Define the associated aggregate loss process as $R(t)=S(t)-c t$, for $t \geq 0$. The probability $\psi(u)$ of ultimate ruin is the probability that the surplus process $C(t)$ ever drops below zero,

$$
\psi(u)=\mathbb{P}\left(\inf _{t \geq 0} C(t)<0\right)=\mathbb{P}\left(\sup _{t \geq 0} R(t)>u\right) .
$$

The maximal aggregate loss $L=\sup _{t \geq 0} R(t)$ can be decomposed as the sum of ladder heights, i.e. as the sum of the amounts by which record lows (here denoted by $L_{1}, L_{2}, \ldots$ ) in the insurer's surplus $C(t)$ appear. Furthermore, the distribution of the $L_{i}(i=1,2, \ldots)$ is given by the integrated tail distribution $F_{L_{i}}(x)=\mu^{-1} \int_{0}^{x}\left(1-F_{X}(z)\right) d z, x>0$. It is well known that $\psi(u)$ is given explicitly by the Pollaczeck-Khinchine formula

$$
\psi(u)=\frac{\theta}{1+\theta} \sum_{k=0}^{\infty}\left(\frac{1}{1+\theta}\right)^{k}\left(1-F_{L_{i}}^{* k}(u)\right),
$$

where $F_{L_{i}}^{* k}$ denotes the $k$-fold convolution of the ladder height distribution (see e.g. Asmussen and Albrecher [Th.IV.2.1] (2009)). The latter expression shows that $L$ is a compound geometric random variable and may be written as $L=\sum_{k=1}^{M} L_{i}$, with $M$ being the number of ladder heights. It is easy to see that $M$ has a geometric distribution with parameter 
$\psi(0)=1 /(1+\theta)($ see, for example Asmussen and Albrecher [Cor.IV.3.1] (2009)). The Laplace transform of (1) is well-known to be

$$
\widehat{\psi}(s)=\int_{0}^{\infty} e^{-s u} \psi(u) d u=\frac{1}{s}-\frac{c-\lambda \mu}{c s-\lambda\left(1-M_{X}(-s)\right),}
$$

where $M_{X}(-s)=\int_{0}^{\infty} e^{-s x} d F_{X}(x)$ is the Laplace-Stieltjes transform of $X$ (cf. Rolski et al. [Th.5.3.3] (2009) or Asmussen and Albrecher [Cor.IV.3.4] (2009)).

For the case when the claim amount distribution has discrete support $\left\{x_{1}, x_{2}, \ldots, x_{m}\right\}$ (with probabilities $p_{1}, p_{2}, \ldots, p_{m}$ ), Schmitter (1990) gave an explicit expression to compute $\psi(u)$ in the form

$$
\psi(u)=1-\frac{\theta}{1+\theta} \sum_{l_{1}, \cdots, l_{m}}\left(-z_{m}\right)^{l_{1}+\cdots l_{m}} \mathrm{e}^{z_{m}} \prod_{j=1}^{m} \frac{p_{j}^{l_{j}}}{l_{j} !},
$$

where $z_{m}=\left(u-l_{1} \cdot x_{1}-\cdots-l_{m} \cdot x_{m}\right)_{+} / \mu \cdot(1+\theta)$ and $z_{+}=\max (z, 0)$.

In the context of the Schmitter problem, 2-point distributions for the claim size play a special role. If $X$ assumes the values $x_{1}$ with probability $p$ and $x_{2}>x_{1}$ with probability $1-p$, then for fixed mean $\mu>0$ and variance $\sigma^{2}>0$ we simply have

$$
\mu=x_{1} \cdot p+x_{2} \cdot(1-p) \text { and } \sigma^{2}+\mu^{2}=x_{1}^{2} \cdot p+x_{2}^{2} \cdot(1-p),
$$

or correspondingly

$$
x_{1}=\mu-\frac{\sigma^{2}}{x_{2}-\mu}, x_{2}=\mu+\frac{\sigma^{2}}{\mu-x_{1}} \text { and } p=\frac{\sigma^{2}}{\sigma^{2}+\left(\mu-x_{1}\right)^{2}},
$$

Notice that $x_{2}$ is increasing in $x_{1}$. Moreover, one has the relationships

$$
\frac{\sigma^{2}}{\mu^{2}+\sigma^{2}} \leq p<1,0 \leq x_{1}<\mu \text {, and } \frac{\mu^{2}+\sigma^{2}}{\mu} \leq x_{2}
$$

(see e.g. Kaas et al. [Ch 10.2] (1994)). If we additionally assume that $X \in[0, b]$, naturally $x_{2} \leq b$, and we have $0 \leq \mu \leq b$ and $0 \leq \sigma^{2} \leq \mu(b-\mu)$. The following two extremal cases will be particularly relevant later. Namely, $X=\left\{0,0^{*}:=\left(\mu^{2}+\sigma^{2}\right) / \mu\right\}$ and so $p=\sigma^{2} /\left(\sigma^{2}+\mu^{2}\right)$ and $X=\left\{b^{*}:=\mu+\sigma^{2} /(\mu-b), b\right\}$. In here, $x^{*}$ denotes the function that assigns to $x$ the unique real number such that the random variable $X=\left\{x, x^{*}\right\}$ has mean $\mu$ and variance $\sigma^{2}$. Note that if $b$ is not bounded, then as $x_{1} \uparrow \mu, p \uparrow 1$ and $x_{2} \rightarrow \infty$; while the probability mass at $x_{2}$ becomes arbitrarily small, it significantly contributes to the variance.

For any non-negative loss variable $X$, the stop-loss premium $\pi_{X}$ is defined by

$$
\pi_{X}(d)=\mathbb{E}\left((X-d)_{+}\right)=\int_{d}^{\infty}\left(1-F_{X}(z)\right) d z, \text { for } d \geq 0 .
$$

Note that there is a one-to-one relation between the integrated tail distribution of $X$ and its stop-loss premium, namely $F_{L_{i}}(z)=1-\pi_{X}(z) / \mu$. One important concept in the theory of risk ordering is the stop-loss order. Concretely, a random variable $X$ is said to be less risky than another random variable $Y$ in stop-loss order $\left(X \leq_{\mathrm{sl}} Y\right)$ if $\pi_{X}(d) \leq \pi_{Y}(d)$ for all retentions $d \geq 0$ (it is equivalent to increasing convex ordering, cf. Shaked and Shanthikumar (2007)). The problem of finding bounds for stop-loss premiums is a classical topic in actuarial science, see for example Bühlmann et al. (1977), 
Kaas and Goovaerts (1986) and Steenackers and Goovaerts (1991). For a study of the relation between stop-loss premiums and their associated ruin probabilities as well as general upper bounds for both stop-loss premiums and ruin probabilities see Cai and Garrido (1998) and the references therein.

A consequence of the above concept is that if for two Cramér-Lundberg risk processes with equal premium per unit of time and claim intensity parameter, but different claim sizes, say $X$ and $Y$, with $X \leq_{\mathrm{sl}} Y$ we have $\psi_{X}(u) \leq \psi_{Y}(u)$ for all $u \geq 0$ (see Kaas et al. [Ch.8.2,Th.2.1] (1994)). Correspondingly, the Schmitter problem may be seen as being reduced to finding extremal distributions in the stop-loss order in the class of random variables in $[0, b]$ with mean $\mu$ and variance $\sigma^{2}$. However, as pointed out in Brockett et al. (1991) there are no extremal distributions in terms of stop-loss order in such a class.

Nevertheless one can construct stop-loss transforms in the corresponding range (bounded or not) with the given mean, but with minimal variance, larger than the given one. For two given moments, the latter is achieved by constructing a polynomial of degree 2 above the function $(X-d)_{+}$which is tangent to this function in 2 points. The abscissas of these points will be the mass points. For a comprehensive description of this construction see Kaas et al. [Ch.10] (1994). In the following we briefly state its main consequences.

For unbounded $X$ with mean $\mu$ and variance $\sigma^{2}$, the maximal stop-loss premium at fixed retention $d$ is attained by a random variable $Z$ with support $\left\{r, r^{*}\right\}$, where $r, r^{*}=d \mp \sqrt{(\mu-d)^{2}+\sigma^{2}}$, and from (3) then $\mathbb{P}(X=r)=\sigma^{2} /\left(\sigma^{2}+(\mu-r)^{2}\right)$. Note that $\left\{r, r^{*}\right\}$ is the 2-point support that has $d$ in the middle. If $X \in[0, b]$, this 2-point distribution still gives an upper bound, but it is no longer always sharp. Theorem 2.3 in Kaas et al. [Ch.10] (1994) provides a sharp upper bound for stop-loss premiums for $X \in[0, b]$. For given retention $d$, the maximal stop-loss premium is attained by the distribution with the mass points

$$
\begin{aligned}
& \left\{0,0^{*}\right\} \quad \text { if } 0 \leq d \leq \frac{1}{2} 0^{*}, \\
& \left\{r, r^{*}\right\} \quad \text { if } \frac{1}{2} 0^{*} \leq d \leq \frac{1}{2}\left(b+b^{*}\right), \\
& \left\{b^{*}, b\right\} \quad \text { if } \frac{1}{2}\left(b+b^{*}\right) \leq d \leq b
\end{aligned}
$$

with the notation introduced before. However, these results do not provide an upper bound for the ruin probability in the Schmitter problem, because it is not the same extremal distribution across all values of $d$, but the latter would be needed to bequeath the dominance in terms of the stop loss premium from the integrated tail to all its convolutions in (1). However, Kaas (1991) showed that if $X$ has lower stop-loss premiums than $Y$ on the interval $[0, u]$, then the same property holds for compound sums with $N$ terms of these random variables respectively, and ruin probabilities with an initial surplus $u$ are lower for $X$ than for $Y$. That is, for values of $u$ smaller than $\frac{1}{2} 0^{*}$, the ruin probability is maximized by the 2-point claim random variable $X=\left\{0,0^{*}\right\}$. Consequently, in terms of the upper bound the Schmitter problem is solved for small values of the initial surplus $u$.

De Vylder and Marceau (1996) and De Vylder et al. (1997b) provided numerical solutions to the problem based on a renewal equation in a discretized risk model. By restricting to lattice distributions, they used the method of linear combinations (see also Kaas et al. [Sec.3] (1992)) to obtain optimal solutions to the problem. They 
noted that for $u \gg b$ the maximal ruin probability was given by the 2-point claim random variable $X=\left\{b^{*}, b\right\}$. In fact, De Vylder et al. (1997a) then proved that there exists a constant $c>0$ such that for all $u \geq c$ the maximal ruin probability is given by that 2-point claim random variable. However, the concrete value of $c$ as well as the optimal result for intermediate values of $u$ seem to still not be settled up to this day.

The minimal stop-loss premium for risks $X$ with mean $\mu$ is given by $(\mu-d)_{+}$for all retentions $d \geq 0$, i.e. it is attained by the defective random variable $Z$ concentrated at $\mu$, implying $Z \leq_{\mathrm{sl}} X$ and therefore $\psi_{Z}(u) \leq \psi_{X}(u)$ for all $u$. However, $Z$ does not fulfill the variance constraint, so that this is not a valid solution to the Schmitter problem. It does provide a general lower bound for its solution though, and for unbounded $X$ the variance constraint can then be satisfied by adding an $\varepsilon(\downarrow 0)$ mass at infinity, see also Asmussen and Albrecher [Cor. IV.8.4] (2009).

\section{Exponentially Distributed Initial Surplus}

Let us now replace the deterministic initial surplus $u$ by a random variable $U$ that has an exponential distribution with parameter $s>0$. The redefined surplus process then is

$$
C^{R}(t)=U+c t-S(t), t \geq 0,
$$

where $c$ and $S(t)$ are defined as in the classical ruin model, and $U$ is independent of $S(t)$. Using the convenient fact that this choice of $U$ simply puts us in the framework of Laplace transforms, due to (2) the ruin probability $\psi_{U}(s):=\mathbb{P}\left(C^{R}(t)<0\right.$ for some $\left.t>0\right)$ is then given by

$$
\psi_{U}(s):=\mathbb{E}(\psi(U))=\int_{0}^{\infty} \psi(u) s e^{-s u} d u=s \cdot \widehat{\psi}(s)=1-s \cdot \frac{c-\lambda \mu}{c s-\lambda\left(1-M_{X}(-s)\right)} .
$$

Since the randomization of the initial surplus corresponds to a probability-weighted averaging over situations with deterministic surplus, it is clear that this step leads to a smoothing of the ruin probability shape. Figure 1 compares the ruin probabilities $\psi(u)$ for deterministic surplus $u=\{1.5,4.5,9.0\}$ and $\theta=0.5$ (the parameters from Kaas [Fig. 1] (1991)) with the corresponding randomized quantities of the same expected initial surplus $\mathbb{E}(U)=1 / s=u$ for 2-point distributions with given mean $\mu=3$ and variance $\sigma^{2}=1$. One observes that the sensitivity w.r.t. the choice of the only free parameter $x_{1}$ is substantially different, and the somewhat curious shape change for increasing $u$ from the classical deterministic case is indeed evened out.

Let us now look at the randomized and extended Schmitter problem

$$
\begin{array}{ll}
\min / \max & \psi_{U}(s) \\
\text { subject to } & \mathbb{E}\left(X^{k}\right)=\mu_{k}, \text { for } k=1,2, \ldots, m
\end{array}
$$

with possibly more than two fixed moments of the claims size distribution. Inspired by Kaas (1991), using the maximal aggregate loss $L$ and assuming that the moments of the claim size are finite, one can express the ruin probability in terms of the claim size moments, namely 

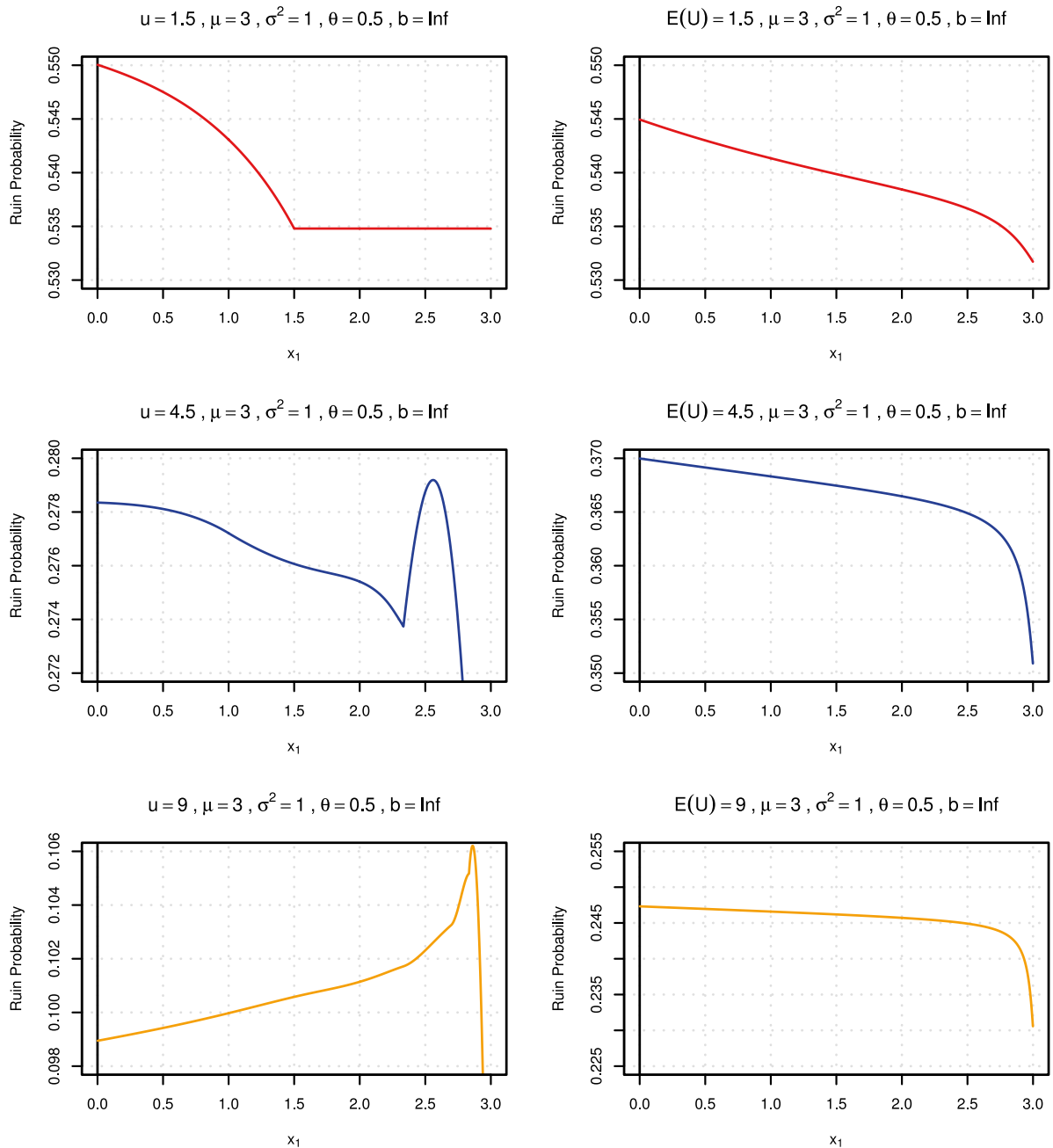

Fig. 1 Ruin probabilities as a function of $x_{1}$ for $\mu=3, \sigma^{2}=1, \theta=0.5$ for the three deterministic surplus levels $u=1.5,4.5,9$ (left column) and the randomized counterpart with the same expected initial surplus level (right column)

$$
\begin{aligned}
\psi_{U}(s) & =\int_{0}^{\infty} \psi(u) s e^{-s u} d u=\sum_{k=0}^{\infty}(-1)^{k} \frac{s^{k+1}}{k !} \int_{0}^{\infty} u^{k} \psi(u) d u \\
& =\sum_{k=0}^{\infty}(-1)^{k} \frac{s^{k+1}}{k !} \int_{0}^{\infty} u^{k} \mathbb{P}(L>u) d u=\sum_{k=1}^{\infty}(-1)^{k-1} \frac{s^{k}}{k !} \mathbb{E}\left(L^{k}\right) \\
& =\sum_{k=1}^{\infty}(-1)^{k-1} \frac{s^{k}}{k !} \mathbb{E}\left(\mathbb{E}\left(\sum_{l_{1}+l_{2}+\cdots+l_{M}=k}\left(\begin{array}{c}
k \\
l_{1}, l_{2}, \ldots, l_{M}
\end{array}\right) \prod_{j=1}^{M} L_{j}^{l_{j}} \mid M\right)\right) .
\end{aligned}
$$

The first four terms of this series are given by 


$$
\begin{aligned}
E(L) & =\mathbb{E}(M) \mathbb{E}\left(L_{1}\right)=\frac{1}{2 \theta \mu} \mathbb{E}\left(X^{2}\right) \\
E\left(L^{2}\right) & =\mathbb{E}(M) \mathbb{E}\left(L_{1}^{2}\right)+\mathbb{E}(M(M-1)) \mathbb{E}^{2}\left(L_{1}\right)=\frac{1}{3 \theta \mu} \mathbb{E}\left(X^{3}\right)+\frac{1}{2 \theta^{2} \mu^{2}} \mathbb{E}\left(X^{2}\right) \\
E\left(L^{3}\right) & =\frac{1}{4 \theta \mu} \mathbb{E}\left(X^{4}\right)+\frac{1}{\theta^{2} \mu^{2}} \mathbb{E}\left(X^{3}\right) \mathbb{E}\left(X^{2}\right)+\frac{3}{4 \theta^{3} \mu^{3}} \mathbb{E}^{3}\left(X^{2}\right) \\
E\left(L^{4}\right) & =\frac{1}{5 \theta \mu} \mathbb{E}\left(X^{5}\right)+\frac{1}{\theta^{2} \mu^{2}} \mathbb{E}\left(X^{4}\right) \mathbb{E}\left(X^{2}\right)+\frac{2}{3 \theta^{2} \mu^{2}} \mathbb{E}^{2}\left(X^{3}\right) \\
& +\frac{1}{6 \theta^{3} \mu^{3}} \mathbb{E}\left(X^{3}\right) \mathbb{E}^{2}\left(X^{2}\right)+\frac{3}{2 \theta^{4} \mu^{4}} \mathbb{E}^{4}\left(X^{2}\right)
\end{aligned}
$$

Hence, if the first $m$ moments of $X$ are given, then one can approximate

$$
\psi_{U}(s) \approx s \mathbb{E}(L)-\frac{s^{2}}{2} \mathbb{E}\left(L^{2}\right)+\cdots+(-1)^{m-1} \frac{s^{m}}{m !} \mathbb{E}\left(L^{m}\right)
$$

and investigate the behavior with respect to the highest moment. For example, for $m=2$

$$
\psi_{U}(s) \approx s \frac{\sigma^{2}+\mu^{2}}{2 \theta \mu}-\frac{s^{2}}{6 \theta \mu} \mathbb{E}\left(X^{3}\right)-s^{2} \frac{\left(\sigma^{2}+\mu^{2}\right)^{2}}{4 \theta^{2} \mu^{2}} .
$$

Therefore, distributions with large third moment will make $\psi_{U}(s)$ small and vice versa. For 2-point distributions, a simple calculation shows that, $\frac{\partial}{\partial x_{1}} \mathbb{E}\left(X^{3}\right)=\sigma^{2}+\left(\frac{\sigma^{2}}{\mu-x_{1}}\right)^{2}>0$ and $\frac{\partial^{2}}{\partial x_{1}^{2}} \mathbb{E}\left(X^{3}\right)=2 \frac{\sigma^{2}}{\left(\mu-x_{1}\right)^{3}}>0$. Thus, for $x_{1} \in[0, \mu)$, its third moment is increasing and convex, so the maximum will be at $x_{1}=0$ and the minimum at $x_{1} \rightarrow \mu$. In fact, for deterministic surplus and 2-point distributions, Kaas (1991) argued that as $\int_{0}^{\infty} \psi(u) d u=\mathbb{E}(L)$ does not depend on $x_{1}$ and $\int_{0}^{\infty} u \psi(u) d u=\mathbb{E}\left(L^{2}\right)$ increases linearly with the third moment of the claim distribution, so that for small $u$, the ruin probability will be large for $x_{1}=0$.

While these considerations are intuitive, from (4) it becomes clear that for the extremal values of the randomized ruin probability it suffices to minimize (maximize) the Laplace transform of the individual claim sizes, i.e. to find extremal random variables in the Laplace transform order. The Laplace transform order has been introduced by Rolski and Stoyan (1976) to compare waiting times in queuing theory. In actuarial science, Denuit (2001) studied both univariate and multivariate versions of the Laplace transform order and gave several actuarial applications. We can now give sharp bounds for the randomized Schmitter problem for two given moments.

Proposition 3.1 Let $X$ be a non-negative random variable with mean $\mu$ and variance $\sigma^{2}$. Then

$$
1-s \cdot \frac{c-\lambda \mu}{c s-\lambda\left(1-e^{-s \mu}\right)} \leq \psi_{U}(s) \leq 1-s \cdot \frac{c-\lambda \mu}{c s-\lambda\left(1-\frac{\sigma^{2}}{\sigma^{2}+\mu^{2}}-\frac{\mu^{2}}{\sigma^{2}+\mu^{2}} e^{-s\left(\mu+\sigma^{2} / \mu\right)}\right)} .
$$

Proof Note that $e^{-s \mu}$ is the Laplace transform of a random variable $Y$ degenerate at $\mu$. Moreover, $\frac{\sigma^{2}}{\sigma^{2}+\mu^{2}}+\frac{\mu^{2}}{\sigma^{2}+\mu^{2}} e^{-s\left(\mu+\sigma^{2} / \mu\right)}$ is the Laplace transform of a random variable $Z$ with mean $\mu$, variance $\sigma^{2}$ and such that $\mathbb{P}(Z=0)=1-\mathbb{P}\left(Z=\left(\mu^{2}+\sigma^{2}\right) / \mu\right)=\frac{\sigma^{2}}{\sigma^{2}+\mu^{2}}$. Therefore, as maximizing the Laplace transform of the individual claim sizes minimizes $\psi_{U}(s)$ and 
vice versa, it suffices to show that $Y \leq_{\mathrm{Lt}} X \leq_{\mathrm{Lt}} Z$. The proof of the latter can be found in Shaked and Shanthikumar [Ch. 5, Theorem 5.A.21] (2007).

It is worth noticing that the distribution maximizing the randomized ruin probability coincides with the 2-point distribution that maximizes the ruin probability under deterministic surplus for small values of $u$. This is rather intuitive, since $\psi_{U}(s)$ is a weighted average of $\psi(u)$ with a lot of weight for small values of $u$.

If more moments of the claim size $X$ in $[0, b]$ are specified, then one can obtain tighter upper and lower bounds for the randomized ruin probability. In view of (4), this reduces to the derivation of bounds for the Laplace transform of $X$ in the moment space $\mathcal{B}_{S}\left([0, b] ; \mu_{1}, \mu_{2}, \ldots, \mu_{m}\right)$ of all risks $X$ with range $[0, b]$ such that $\mathbb{E}\left(X^{k}\right)=\mu_{k}$ for $k=1,2, \ldots, m$. Fortunately, our context fits exactly into the framework of Denuit et al. $(1999,1998)$ who constructed lower and upper stochastic bounds for a given set of risks using $m$-convex stochastic orders. More precisely, consider the class $\mathcal{M}_{m-c x}$ of all functions $\phi:[0, b] \rightarrow \mathbb{R}$ whose $(m+1)$-th derivative $\phi^{(m+1)}(x)$ exists and is non-negative, for all $x \in[0, b]$, or which are limits of sequences of functions whose $(m+1)$-th derivative is continuous and non-negative on $[0, b]$. Define the partial order relation $\leq_{m-c x}$ among elements in $\mathcal{B}_{S}$ as

$$
X \leq_{m-c x} Y \text { if and only if } \mathbb{E}(\phi(X)) \leq \mathbb{E}(\phi(Y)) \text { for all functions } \phi \in \mathcal{M}_{m-c x},
$$

provided the expectations exists. It is then possible to determine two discrete risks $X_{\min }^{(m)}$ and $X_{\max }^{(m)}$, in $\mathcal{B}_{S}\left([0, b] ; \mu_{1}, \mu_{2}, \ldots, \mu_{m}\right)$ with probability masses depending on the moment set $\left(\mu_{1}, \mu_{2}, \ldots, \mu_{m}\right)$ such that

$$
X_{\min }^{(m)} \leq_{m-c x} X \leq_{m-c x} X_{\max }^{(m)} \text { for all } X \in \mathcal{B}_{S} .
$$

Explicit descriptions for the distribution functions of the extrema up to $m=4$ are obtained in Denuit et al. [Table 1, Table 2] (1999). Moreover, the latter reference also provided the extrema with respect to the order $\leq_{m-c x}$ when not only the first $m-1$ moments and the support are given, but also when the density function of $X$ is known to be unimodal with a known mode. ${ }^{1}$

Proposition 3.2 Let $X \in[0, b], b>0$, with moments $\left(\mu_{1}, \mu_{2}, \ldots, \mu_{m}\right)$. Then,

$$
\begin{aligned}
& M_{X_{\max }^{(m)}}(-s) \leq M_{X}(-s) \leq M_{X_{\min }^{(m)}}(-s), \text { for } \mathrm{m}+1 \text { odd } \\
& M_{X_{\min }^{(m)}}(-s) \leq M_{X}(-s) \leq M_{X_{\max }^{(m)}}(-s), \text { for } \mathrm{m}+1 \text { even, }
\end{aligned}
$$

which can then be translated to bounds for $\psi_{U}(s)$.

Proof Since $\phi(x)=(-1)^{m+1} e^{-s x}$ belongs to $\mathcal{M}_{m-c x}$ for $s>0$, the claim follows from (6) and (7).

The bounds for the Laplace transform using $m$-convex risks were already described in Denuit et al. (2000), extending earlier works of Eckberg (1977), Whitt (1983) and Lefèvre et al. (1986). In particular, Eckberg (1977) derived bounds for the Laplace

\footnotetext{
1 Note that in this paper we use the notation $\leq_{m-c x}$ to denote $m$-convexity whenever the first $m$ moments are available and not $(m-1)$ as it is standard in the $m$-convex risk literature.
} 
transform up to the third moment using the theory of Chebychev systems and applied the bounds to problems in queuing and traffic theory. Moreover, the latter reference provides bounds for the case where no upper bound is known. We would also like to mention that, closely related to the theory of $m$-convex stochastic orders, using MarkovKrein theory and the theory of Chebychev systems, Brockett and Cox $(1984,1985)$ obtained similar upper and lower bounds for the expected value of a function of some random variable with given moments. Also, De Vylder (1982, 1983), De Vylder and Goovaerts (1982), Kaas and Goovaerts (1986) and Hürlimann (1998) examined related bounding problems.

Using (8) we can give explicit bounds for the ruin probability with exponentially distributed initial surplus in terms of the given parameters. For reference, we restate here the respective bounds given in Denuit et al. [Table 1, Table 2] (1999)) in terms of ruin probabilities when up to 4 moments of $X$ are given:

Case $m=1$. If $\mu_{1}$ is given, then $X_{\min }^{(1)}$ is a random variable degenerate at $\mu_{1}$, and

$$
X_{\max }^{(1)}= \begin{cases}0 & \text { with } p=1-\frac{\mu_{1}}{b}, \\ b & \text { with } 1-p=\frac{\mu_{1}}{b} .\end{cases}
$$

Therefore,

$$
\begin{aligned}
& \psi_{U}^{\min }(s)=1-s \cdot \frac{c-\lambda \mu_{1}}{c s-\lambda\left(1-e^{-s \mu_{1}}\right)}, \\
& \psi_{U}^{\max }(s)=1-s \cdot \frac{c-\lambda \mu_{1}}{c s-\frac{\lambda \mu_{1}}{b}\left(1-e^{-s b}\right)} .
\end{aligned}
$$

Case $m=2$. If $\mu_{1}$ and $\mu_{2}$ are given, then

$$
X_{\min }^{(2)}=\left\{\begin{array}{ll}
0 \quad \text { with } p=1-\frac{\mu_{1}^{2}}{\mu_{2}}, \\
\frac{\mu_{2}}{\mu_{1}} \quad \text { with } 1-p=\frac{\mu_{1}^{2}}{\mu_{2}},
\end{array} \quad X_{\max }^{(2)}= \begin{cases}\frac{b \mu_{1}-\mu_{2}}{b-\mu_{1}} & \text { with } p=\frac{\left(b-\mu_{1}\right)^{2}}{\left(b-\mu_{1}\right)^{2}+\mu_{2}-\mu_{1}^{2}}, \\
b & \text { with } 1-p=\frac{\mu_{2}-\mu_{1}^{2}}{\left(b-\mu_{1}\right)^{2}+\mu_{2}-\mu_{1}^{2}} .\end{cases}\right.
$$

In this case, it can be seen that

$$
\begin{aligned}
& \psi_{U}^{\min }(s)=1-s \cdot \frac{c-\lambda \mu_{1}}{c s-\lambda\left(1-\frac{\left(b-\mu_{1}\right)^{2}}{\sigma^{2}+\left(b-\mu_{1}\right)^{2}} e^{-s\left(\mu_{1}-\sigma^{2} /\left(b-\mu_{1}\right)\right)}-\frac{\sigma^{2}}{\sigma^{2}+\left(b-\mu_{1}\right)^{2}} e^{-s b}\right)}, \\
& \psi_{U}^{\max }(s)=1-s \cdot \frac{c-\lambda \mu_{1}}{c s-\frac{\lambda \mu_{1}^{2}}{\sigma^{2}+\mu_{1}^{2}}\left(1-e^{-s\left(\mu_{1}+\sigma^{2} / \mu_{1}\right)}\right)} .
\end{aligned}
$$

Note that for $b \rightarrow \infty$ the above expressions indeed converge to the bounds given in Proposition 3.1.

Case $m=3$. If $\mu_{1}, \mu_{2}$ and $\mu_{3}$ are given, then 


$$
\begin{aligned}
& X_{\min }^{(3)}=\left\{\begin{array}{l}
r_{+}=\frac{\mu_{3}-\mu_{1} \mu_{2}+\sqrt{\left(\mu_{3}-\mu_{1} \mu_{2}\right)^{2}-4 \sigma^{2}\left(\mu_{1} \mu_{3}-\mu_{2}^{2}\right)}}{2 \sigma^{2}} \quad \text { with } p=\frac{\mu_{1}-r_{-}}{r_{+}-r_{-}}, \\
r_{-}=\frac{\mu_{3}-\mu_{1} \mu_{2}-\sqrt{\left(\mu_{3}-\mu_{1} \mu_{2}\right)^{2}-4 \sigma^{2}\left(\mu_{1} \mu_{3}-\mu_{2}^{2}\right)}}{2 \sigma^{2}} \text { with } 1-p=1-\frac{\mu_{1}-r_{-}}{r_{+}-r_{-}},
\end{array}\right. \\
& X_{\max }^{(3)}= \begin{cases}0 & \text { with } p_{3}=1-p_{1}-p_{2}, \\
\frac{\mu_{3}-b \mu_{2}}{\mu_{2}-b \mu_{1}} & \text { with } p_{1}=\frac{\left(\mu_{2}-b \mu_{1}\right)^{3}}{\left(\mu_{3}-b \mu_{2}\right)\left(\mu_{3}-2 b \mu_{2}+b^{2} \mu_{1}\right)}, \\
b & \text { with } p_{2}=\frac{\mu_{1} \mu_{3}-\mu_{2}^{2}}{b\left(\mu_{3}-2 b \mu_{2}+b^{2} \mu_{1}\right)} .\end{cases}
\end{aligned}
$$

Then, the bounds for the ruin probability are given by

$$
\begin{gathered}
\psi_{U}^{\min }(s)=1-s \cdot \frac{c-\lambda \mu_{1}}{c s-\lambda\left(1-\left(1-\frac{\mu_{1}-r_{-}}{r_{+}-r_{-}}\right) e^{-s r_{-}}-\left(\frac{\mu_{1}-r_{-}}{r_{+}-r_{-}}\right) e^{-s r_{+}}\right)}, \\
\psi_{U}^{\max }(s)=1-s \cdot \frac{c-\lambda \mu_{1}}{c s-\lambda\left(p_{1}\left(1-e^{-s \frac{\mu_{3}-b \mu_{2}}{\mu_{2}-b \mu_{1}}}\right)+p_{2}\left(1-e^{-s b}\right)\right)} .
\end{gathered}
$$

Case $m=4$. If $\mu_{1}$ and up to $\mu_{4}$ are given, then

$$
\begin{aligned}
& X_{\min }^{(4)}= \begin{cases}0 & \text { with } 1-p_{+}-p_{-}, \\
t_{+}=\frac{\mu_{1} \mu_{4}-\mu_{2} \mu_{3}+\sqrt{\left(\mu_{1} \mu_{4}-\mu_{2} \mu_{3}\right)^{2}-4\left(\mu_{1} \mu_{3}-\mu_{2}^{2}\right)\left(\mu_{2} \mu_{4}-\mu_{3}^{2}\right)}}{2\left(\mu_{1} \mu_{3}-\mu_{2}^{2}\right)} & \text { with } p_{+}=\frac{\mu_{2}-t_{-} \mu_{1}}{t_{+}\left(t_{+}-t_{-}\right)}, \\
t_{-}=\frac{\mu_{1} \mu_{4}-\mu_{2} \mu_{3}-\sqrt{\left(\mu_{1} \mu_{4}-\mu_{2} \mu_{3}\right)^{2}-4\left(\mu_{1} \mu_{3}-\mu_{2}^{2}\right)\left(\mu_{2} \mu_{4}-\mu_{3}^{2}\right)}}{2\left(\mu_{1} \mu_{3}-\mu_{2}^{2}\right)} & \text { with } p_{-}=\frac{\mu_{2}-t_{+} \mu_{1}}{t_{-}\left(t_{-}-t_{+}\right)}\end{cases} \\
& X_{\max }^{(4)}= \begin{cases}z_{+}=\frac{\left(\mu_{1}-b\right)\left(\mu_{4}-b \mu_{3}\right)-\left(\mu_{2}-b \mu_{1}\right)\left(\mu_{3}-b \mu_{2}\right)+\sqrt{\rho}}{2\left(\left(\mu_{1}-b\right)\left(\mu_{3}-b \mu_{2}\right)-\left(\mu_{2}-b \mu_{1}\right)^{2}\right)} & \text { with } q_{+}=\frac{\mu_{2}-\left(b+z_{-}\right) \mu_{1}+b z_{-}}{\left(z_{+}-z_{-}\right)\left(z_{+}-b\right)} \\
z_{-}=\frac{\left(\mu_{1}-b\right)\left(\mu_{4}-b \mu_{3}\right)-\left(\mu_{2}-b \mu_{1}\right)\left(\mu_{3}-b \mu_{2}\right)-\sqrt{\rho}}{2\left(\left(\mu_{1}-b\right)\left(\mu_{3}-b \mu_{2}\right)-\left(\mu_{2}-b \mu_{1}\right)^{2}\right)} & \text { with } q_{-}=\frac{\mu_{2}-\left(b+z_{+}\right) \mu_{1}+b z_{+}}{\left(z_{-}-z_{+}\right)\left(z_{-}-b\right)} \\
b & \text { with } 1-q_{+}-q_{-} \cdot\end{cases}
\end{aligned}
$$

Here,

$$
\begin{aligned}
\rho:= & \left(\left(\mu_{1}-b\right)\left(\mu_{4}-b \mu_{3}\right)-\left(\mu_{2}-b \mu\right)\left(\mu_{3}-b \mu_{2}\right)\right)^{2} \\
& \quad-4\left(\left(\mu_{1}-b\right)\left(\mu_{3}-b \mu_{2}\right)-\left(\mu_{2}-b \mu_{1}\right)^{2}\right)\left(\left(\mu_{2}-b \mu_{1}\right)\left(\mu_{4}-b \mu_{3}\right)-\left(\mu_{3}-b \mu_{2}\right)^{2}\right)
\end{aligned}
$$

As can easily be verified,

$$
\begin{aligned}
& \psi_{U}^{\min }(s)=1-s \cdot \frac{c-\lambda \mu_{1}}{c s-\lambda\left(1-q_{+} e^{-s z_{+}}-q_{-} e^{-s z_{-}}-\left(1-q_{+}-q_{-}\right) e^{-s b}\right)}, \\
& \psi_{U}^{\max }(s)=1-s \cdot \frac{c-\lambda \mu_{1}}{c s-\lambda\left(p_{+}\left(1-e^{-s t_{+}}\right)+p_{-}\left(1-e^{-s t_{-}}\right)\right)} .
\end{aligned}
$$




\subsection{Numerical Illustrations}

De Vylder [Sec.II, Ch.3] (1996) gives conditions for the class of all vectors $\left(\mu_{1}, \mu_{2}, \ldots, \mu_{m}\right) \in \mathbb{R}^{m}$ such that $\mathcal{B}_{S}\left([0, b] ; \mu_{1}, \mu_{2}, \ldots, \mu_{m}\right)$ is not empty. In Denuit et al. [Sec.4.1] (1999), this class of all possible moment sequences is denoted by $\mathcal{D}_{m}([0, b])$. Moreover, they provided expressions for the topological interior, $\mathcal{D}_{m} \circ([0, b])$, of $\mathcal{D}_{m}([0, b])$ up to $m=4$. For completeness we cite the three cases relevant for our applications here, namely:

$$
\begin{aligned}
\mathcal{D}_{1} \circ([0, b]) & =\left\{\mu_{1} \in \mathbb{R} \mid 0<\mu_{1}<b\right\}, \\
\mathcal{D}_{2} \circ([0, b]) & =\left\{\left(\mu_{1}, \mu_{2}\right) \in \mathbb{R}^{2} \mid \mu_{1} \in \mathcal{D}_{1} \circ([0, b]) \text { and } \mu_{1}^{2}<\mu_{2}<\mu_{1} b\right\}, \\
\mathcal{D}_{3} \circ([0, b]) & =\left\{\left(\mu_{1}, \mu_{2}, \mu_{3}\right) \in \mathbb{R}^{3} \mid\left(\mu_{1}, \mu_{2}\right) \in \mathcal{D}_{2} \circ([0, b])\right. \text { and } \\
& \left.\frac{\sigma^{2}}{\mu_{1}}\left(\sigma^{2}-\mu_{1}^{2}\right)-2 \mu_{1}^{3}+3 \mu_{1} \mu_{2}<\mu_{3}<\left(b-\mu_{1}\right) \sigma^{2}-\frac{\sigma^{4}}{b-\mu_{1}}-2 \mu_{1}^{3}+3 \mu_{1} \mu_{2}\right\} .
\end{aligned}
$$

Figure 2 depicts the sharp bounds for the ruin probability with $b=100, \theta=0.5$ and $s=2 / 5$, i.e. $\mathbb{E}(U)=2.5$. The upper left figure shows the bounds for $\mu_{1}=3.95$ as a function of $\mu_{2}$ satisfying $\left(\mu_{1}, \mu_{2}\right) \in \mathcal{D}_{2} \circ([0, b])$. For this case, we also know the upper bound solution of the Schmitter problem with deterministic surplus and we can compare the two. It turns out that the upper bounds of the randomized and the deterministic case are remarkably close. The upper right figure shows the sharp ruin probability bounds for three given moments as a function of $\mu_{3}$ satisfying $\left(3.95,48.62, \mu_{3}\right) \in \mathcal{D}_{3} \circ([0, b])$. As remarked in
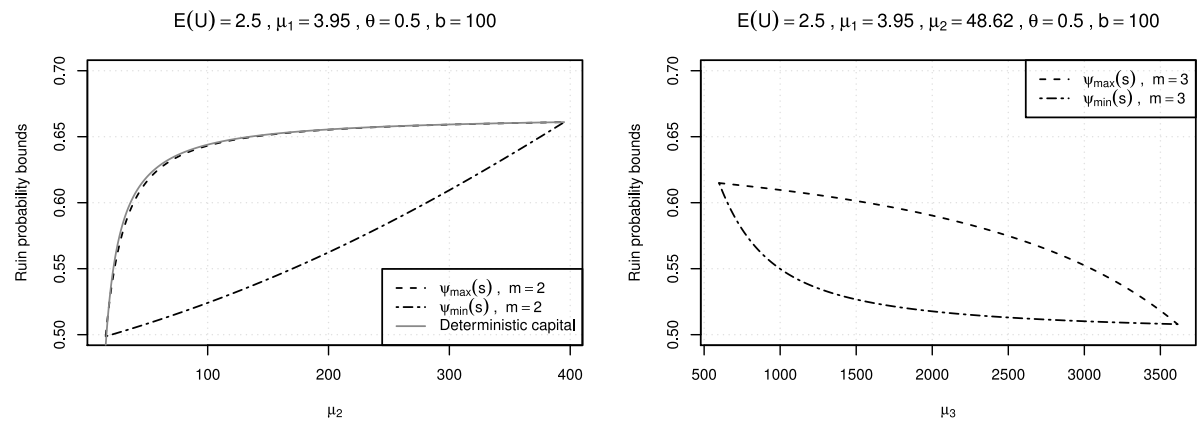

$E(U)=2.5, \mu_{1}=3.95, \mu_{2}=48.62, \mu_{3}=1090.95, \theta=0.5, b=100$

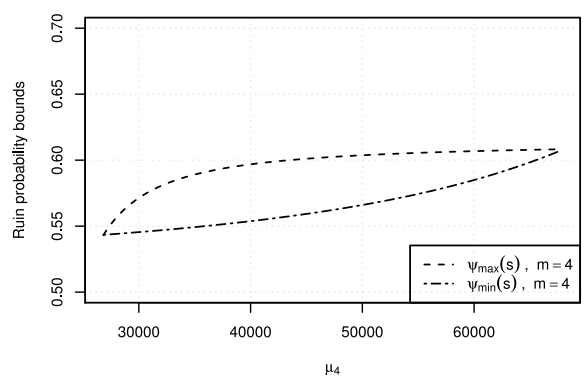

Fig. 2 Sharp bounds for the randomized ruin probability $\psi_{U}(2 / 5)$, considered as a function of $\mu_{2}, \mu_{3}$ and $\mu_{4}$ respectively 
the previous section, once sees that the ruin probability decreases with increasing third moment. As expected, the bounds are tighter as the knowledge of the second moment is incorporated. Finally, the graph at the bottom depicts the bounds of the generalized randomized Schmitter problem for given four moments of $X$ as a function of $\mu_{4}$, leading to yet tighter bounds. Note that in this numerical illustration the values of the first three moments were selected in such a way that one finds a feasible set of distribution parameters for all of the distributions in the following numerical illustration.

In order to illustrate the performance of the bounds and how they improve with the addition of moment information, we explicitly calculate $\psi_{U}(s)$ for some chosen claim size distribution in each case, suitably truncated so that it fits into the model setup:

- Case $m=1$. Truncated Exponential $(\lambda)$ model with distribution function given by

$$
F_{X}(x)=\frac{1-e^{-\lambda x}}{1-e^{-\lambda b}}, 0<x \leq b, \lambda>0
$$

and Laplace transform

$$
M_{X}(-s)=\frac{\lambda}{\lambda+s} \frac{1-e^{-(\lambda+s) b}}{1-e^{-\lambda b}} .
$$

- $\quad$ Case $m=2$.

- Truncated Gamma $(\alpha, \beta)$ model with distribution function

$$
F_{X}(x)=\frac{\gamma(\alpha, \beta x)}{\gamma(\alpha, \beta b)}, 0<x \leq b, \alpha, \beta>0,
$$

and Laplace transform

$$
M_{X}(-s)=\left(\frac{\beta}{\beta+s}\right)^{\alpha} \frac{\gamma(\alpha,(\beta+s) b)}{\gamma(\alpha, \beta b)},
$$

where $\gamma(\alpha, x)=\int_{0}^{x} z^{\alpha-1} e^{-z} d z$ is the lower incomplete gamma function.

- Truncated US-Pareto $(\alpha, \eta)$ (Lomax) model with distribution function

$$
F_{X}(x)=\frac{1-\left(\frac{\eta}{\eta+x}\right)^{\alpha}}{1-\left(\frac{\eta}{\eta+b}\right)^{\alpha}}, 0 \leq x \leq b, \alpha, \eta>0,
$$

and Laplace transform

$$
M_{X}(-s)=\frac{\alpha(\eta s)^{\alpha} e^{\eta s}}{1-\left(\frac{\eta}{\eta+b}\right)^{\alpha}}(\Gamma(-\alpha, \eta s)-\Gamma(-\alpha,(\eta+b) s)),
$$

where $\Gamma(\alpha, x)=\int_{x}^{\infty} z^{\alpha-1} e^{-z} d z$ is the upper incomplete gamma function.

- Case $m=3$. Truncated generalized Gamma $(\alpha, \beta, \tau)$ model with density and distribution function given by

$$
f_{X}(x)=\frac{\tau x^{\alpha \tau-1} \beta^{-\alpha \tau} e^{-(x / \beta)^{\tau}}}{\gamma\left(\alpha,(b / \beta)^{\tau}\right)}, F_{X}(x)=\frac{\gamma\left(\alpha,(x / \beta)^{\tau}\right)}{\gamma\left(\alpha,(b / \beta)^{\tau}\right)}, 0<x \leq b, \alpha, \beta \tau>0,
$$


and Laplace transform

$$
M_{X}(-s)=\sum_{k=0}^{\infty} \frac{(-\beta s)^{k}}{k !} \frac{\gamma\left(\alpha+k / \tau,(b / \beta)^{\tau}\right)}{\gamma\left(\alpha,(b / \beta)^{\tau}\right)} .
$$

In each case, the distribution parameters were determined using the method of moments for the given moment values set in the example above. For further details on claim size distributions and truncation see, for example, Albrecher et al. [Sec.3.3 \& Ch.4] (2017).

The results are given in Fig. $3 \mathrm{a}$ where the exact ruin probabilities obtained using (4) together with the general bounds are plotted as a function of the expected initial surplus $\mathbb{E}(U)=1 / s$ for the same set of parameters as above. In particular, $b$ was selected in such a way that no strong truncation effect is present in the distributions. One sees that, for fixed $\mu_{1}$ only, the truncated exponential case is nicely between the sharp bounds. However, these bounds are very wide. When information about the second moment of $X$ is included, the tightness of the bounds improves significantly. From (5), one would expect that to be the case only for small values of $s$ where information about the first two moments provides a good approximation for the ruin probability. However, we can see that even for large values of $s$ the improvement is considerable. The tightness of the interval for possible ruin probabilities becomes even more remarkable when the first three moments are fixed. This illustrates that in the context of ruin probabilities, the knowledge of the first few moments of the claim size distribution already provides a very accurate approximation. In a broader statistical context, for an account on reconstructions of arbitrary distributions from given moments, see e.g. Mnatsakanov (2008). Finally, for recent progress on the general probability level concerning criteria of moment-determinacy of distributions, see Yarovaya et al. (2020).

Remark 3.1 All results from this section can easily be generalized to the case where the initial surplus is assumed to be a mixture of exponential random variables. Indeed, consider a random initial surplus $O$ with density

$$
f_{O}(u)=\sum_{i=1}^{n} q_{i} k_{i} e^{-k_{i} \cdot u},
$$

with $0<q_{i}<1, \sum_{i=1}^{n} q_{i}=1$ and $k_{i}>0$ for $i=1, \ldots, n$. Then

$$
\begin{aligned}
\psi_{O}:=\mathbb{E}(\psi(O)) & =\sum_{i=1}^{n} q_{i} \int_{0}^{\infty} \psi(u) k_{i} e^{-k_{i} u} d u=\sum_{i=1}^{n} q_{i} \cdot \psi_{U}\left(k_{i}\right)=\sum_{i=1}^{n} q_{i} k_{i} \cdot \widehat{\psi}\left(k_{i}\right) \\
& =1-\sum_{i=1}^{n} q_{i} k_{i} \frac{c-\lambda \mu}{c k_{i}-\lambda\left(1-M_{X}\left(-k_{i}\right)\right)}
\end{aligned}
$$

Since Proposition 3.2 applies to any value $s>0$, for every given set of $m$ moment constraints and all $k_{i}>0, i=1, \ldots, n$, we obtain $\psi_{U}^{\min }\left(k_{i}\right) \leq \psi_{U}\left(k_{i}\right) \leq \psi_{U}^{\max }\left(k_{i}\right)$. Therefore,

$$
\sum_{i=1}^{n} q_{i} \psi_{U}^{\min }\left(k_{i}\right) \leq \psi_{O} \leq \sum_{i=i}^{n} q_{i} \psi_{U}^{\max }\left(k_{i}\right) .
$$

Consequently, when $U$ is a mixture of exponential random variables, the lower and upper bounds for the expected ruin probability are linear combinations of the respective 
$\mu_{1}=3.95, \theta=0.5, b=100$

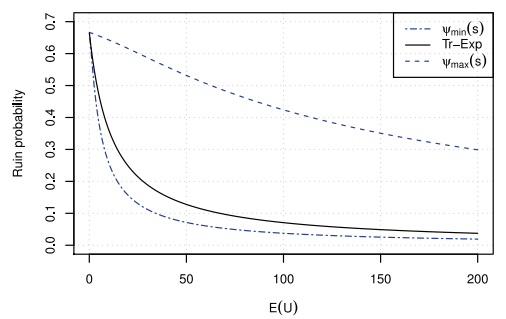

$\mu_{1}=3.95, \mu_{2}=48.62, \theta=0.5, b=100$

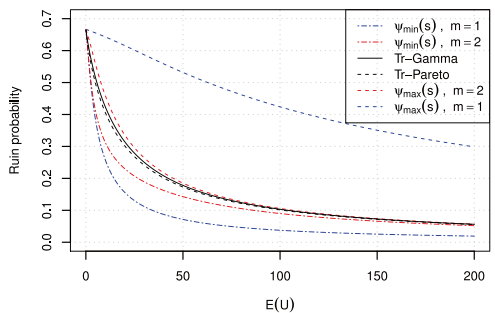

$\mu_{1}=3.95, \mu_{2}=48.62, \mu_{3}=1090.95, \theta=0.5, b=100$

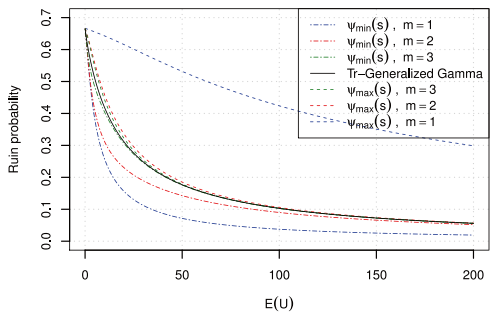

(A) Ruin probabilities $\psi_{U}(s)$ as a function of the expected initial surplus. Top left: Bounds for $\psi_{U}(s)$ together with the case of truncated exponential claims for given $\mu_{1}$. Top right: Bounds for $\psi_{U}(s)$ together with the case of truncated gamma and truncated US-Pareto claims for given $\mu_{1}$ and $\mu_{2}$. Bottom: Bounds for $\psi_{U}(s)$ together with truncated generalized gamma claims when $\mu_{1}, \mu_{2}$ and $\mu_{3}$ are given.
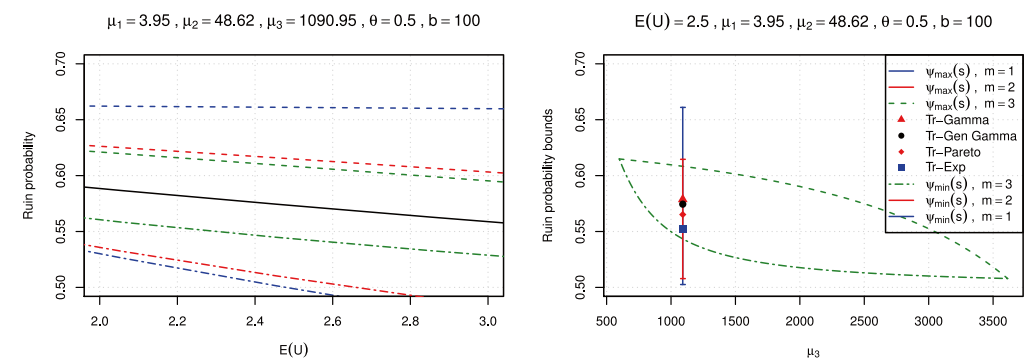

(B) Left: Magnification around $\mathbb{E}(U)=2.5$ of the ruin probabilities $\psi_{U}(s)$ and the upper/lower bounds in Figure 3a (bottom). Right: Bounds for $\psi_{U}(2 / 5)$, considered as a function of $\mu_{3}$ (Figure 2 (top right)), with a vertical bar at $\mu_{3}=1090.95$ summarizing the upper/lower bounds and ruin probabilities in Figure $3 \mathrm{a}$ at $\mathbb{E}(U)=2.5$.

Fig. 3 Numerical illustration for bounds of the ruin probability under exponentially distributed initial surplus

upper and lower bounds for the ruin probability with exponentially distributed initial surplus.

Note that for obtaining these sharp bounds, one still needed to reduce the expressions to purely exponential components so that the bounds on Laplace transforms can be used. For more general assumptions on $U$ (like a general phase-type distribution) that link cannot be carried over in such a direct way. In the next section, we will, however, study the case of $\operatorname{Erlang}(k)$ distributed $U$ in more detail, which is of particular interest, as for large $k$ a deterministic initial surplus level can be approximated. 


\section{Erlang Distributed Initial Surplus}

A natural extension of exponentially distributed random surplus is now to consider Erlang distributed initial surplus. Concretely, consider $U$ to be an $\operatorname{Erlang}(k, s)$ random variable $E_{k}$ with density

$$
f_{E_{k}}(x)=\frac{1}{(k-1) !} s^{k} x^{k-1} e^{-s x} \text { for } k \geq 1, s>0, x>0,
$$

We then get

$$
\psi_{E}(k, s):=\mathbb{E}\left(\psi\left(E_{k}\right)\right)=\int_{0}^{\infty} \psi(u) \frac{s^{k}}{(k-1) !} u^{k-1} e^{-s u} d u=1+\frac{(-s)^{k}}{(k-1) !} \frac{\partial^{(k-1)}}{\partial s^{(k-1)}} \widehat{\phi}(s) .
$$

Here $\hat{\phi}(s)=1 / s-\widehat{\psi}(s)$ denotes the Laplace transform of the survival probability of the classical Cramér-Lundberg risk process and we observe that its derivatives w.r.t. the Laplace argument lead to an explicit expression for the case of random Erlang-distributed initial surplus. We focus here on the classical Schmitter setting with fixed mean and variance of the claim size distribution. In Fig. 4 we depict the ruin probabilities for $\operatorname{Erlang}(k, s)$ distributed initial surplus for 2-point distributions as a function of $x_{1}$ for a given mean and variance, for two expected surplus levels.

In contrast to the exponential case $(k=1)$, there is unfortunately no direct relation between the optimization problem and the minimization (maximization) of the Laplace transform of the ruin probability. What we obtain is in fact an expression in terms of its $(k-1)$-th derivative $\left(\right.$ with $\partial^{(0)} / \partial s^{(0)} \hat{\phi}(s)=\hat{\phi}(s)$ ). For example, for $k=2$ we get

$$
\begin{aligned}
\psi_{E}(2, s) & =1+s^{2} \frac{\partial}{\partial s} \hat{\phi}_{u}(s)=1+s^{2} \frac{\partial}{\partial s} \frac{c-\lambda \mu}{c s-\lambda\left(1-M_{X}(-s)\right)} \\
& =1-s^{2} \frac{c+\lambda \frac{\partial}{\partial s} M_{X}(-s)}{c-\lambda \mu}\left(\frac{c-\lambda \mu}{c s-\lambda\left(1-M_{X}(-s)\right)}\right)^{2}, \quad s>0 .
\end{aligned}
$$

Thus, for a fixed parameter $s$, in order to maximize our ruin probability, we need to minimize an expression that depends on both the Laplace transform of $X$ and its first derivative.
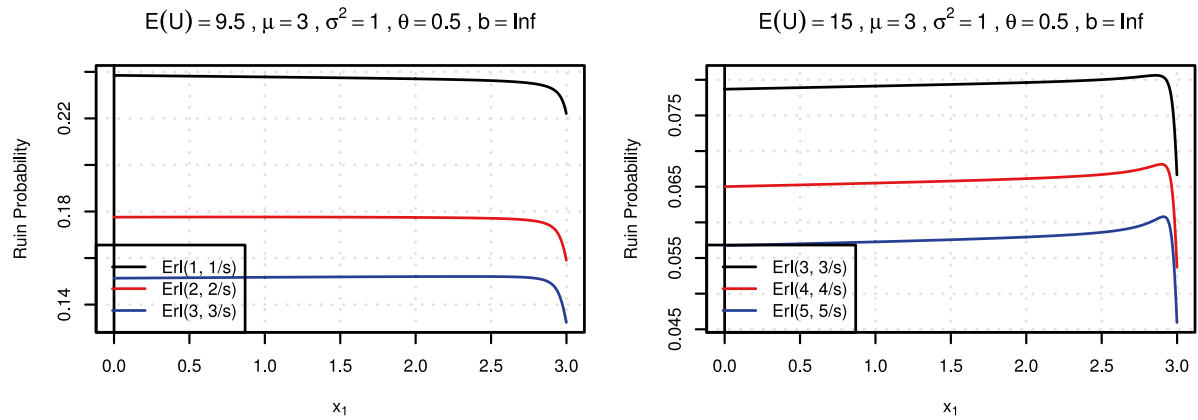

Fig. 4 Ruin probabilities for $\operatorname{Erlang}(k, k / u)$ distributed surplus as a function of $x_{1}$ for $\mu=3, \sigma^{2}=1, \theta=0.5$ and $\mathbb{E}(U)$ as specified 
Since the variance of a Erlang distribution goes to 0 as $k \rightarrow \infty$, one particular motivation to consider Erlang distributed initial surplus is as a tool to approximate the case of deterministic initial surplus, as in fact one has $\psi_{E}(k, s) \rightarrow \psi(u)$ as $k \rightarrow \infty$. The approximation $\psi(u) \approx \psi_{E}(k, s)$, or Erlang smoothing, was considered in Asmussen et al. (2002) as a numerical scheme to approximate the finite horizon ruin probability by replacing the deterministic time horizon $T$ by an "standarized" Erlang $(k, k / T)$ random variable, which for $k \rightarrow \infty$ becomes exact (see Asmussen and Albrecher [Ch.IX.8] (2009) for a more general discussion, as well as Stanford et al. (2005), Carr (1998) and Kyprianou and Pistorius (2003) for applications of this approach to other fields). Concerning the convergence rate with increasing $k$, for our context of random initial surplus one can adapt Theorem 6 of Asmussen et al. (2002) in a straightforward way to obtain the following result:

Proposition 4.1 Let $u>0$ be the expected initial surplus and let $E_{k}$ denote the Erlang distribution with shape parameter $k$ and mean $u$. Then $\psi_{E}(k, s) \rightarrow \psi(u)$ as $k \rightarrow \infty$. More precisely, for some constant $C$

$$
\psi_{E}(k, k / u)=\psi(u)+\frac{C}{k}+O\left(k^{-2}\right) .
$$

As already suggested in Asmussen et al. (2002), a further improvement of accuracy for fixed $k$ can be obtained by Richardson extrapolation. This is a general method (see e.g. Press et al. (2007) for details) for computing an abstract quantity $y$ (it could be an integral, a derivative, etc.) accurately using a sequence $y_{k} \rightarrow y$ for which the convergence rate is known,

$$
y_{k}=y+\frac{c_{1}}{k}+\frac{c_{2}}{k^{1+\epsilon}}+\ldots,
$$

where $c_{1}$ is typically unknown but can be eliminated. In fact, setting $\tilde{y}_{k}=(k+1) y_{k+1}-k y_{k}$, we get that $\tilde{y}_{k} \rightarrow y$ and one obtains an improved approximation of convergence rate $O\left(k^{-1-\epsilon}\right)$.

Translated into our context, we then get

$$
\psi(u) \approx(k+1) \psi_{E}(k+1,(k+1) / u)-k \psi_{E}(k, k / u),
$$

with an error rate of order $1 / k^{2}$.

For an illustration of the method, consider the same example as in Fig. 1, namely the set of 2-point distributions with mean $\mu=3$, variance $\sigma^{2}=1$, and safety loading $\theta=0.5$. Figure 5 shows the results of the approximation. One observes that the approximation of the deterministic case via the randomized initial surplus is quite satisfactory already for $k=11$. The numerical approximation works well even for intermediate values of the initial surplus for which the ruin probability (and its kink) is difficult to approximate. In order to also reproduce the particular shape of that curve, higher values of $k$ are however needed. It is worth to note the tremendous improvement when employing Richardson extrapolation for larger values of $u$ (cf. the graph for $u=9$ ).

Remark 4.1 Analogous to the exponential initial surplus case, one can obtain an expression for the ruin probability in terms of moments of $L$. Concretely, 

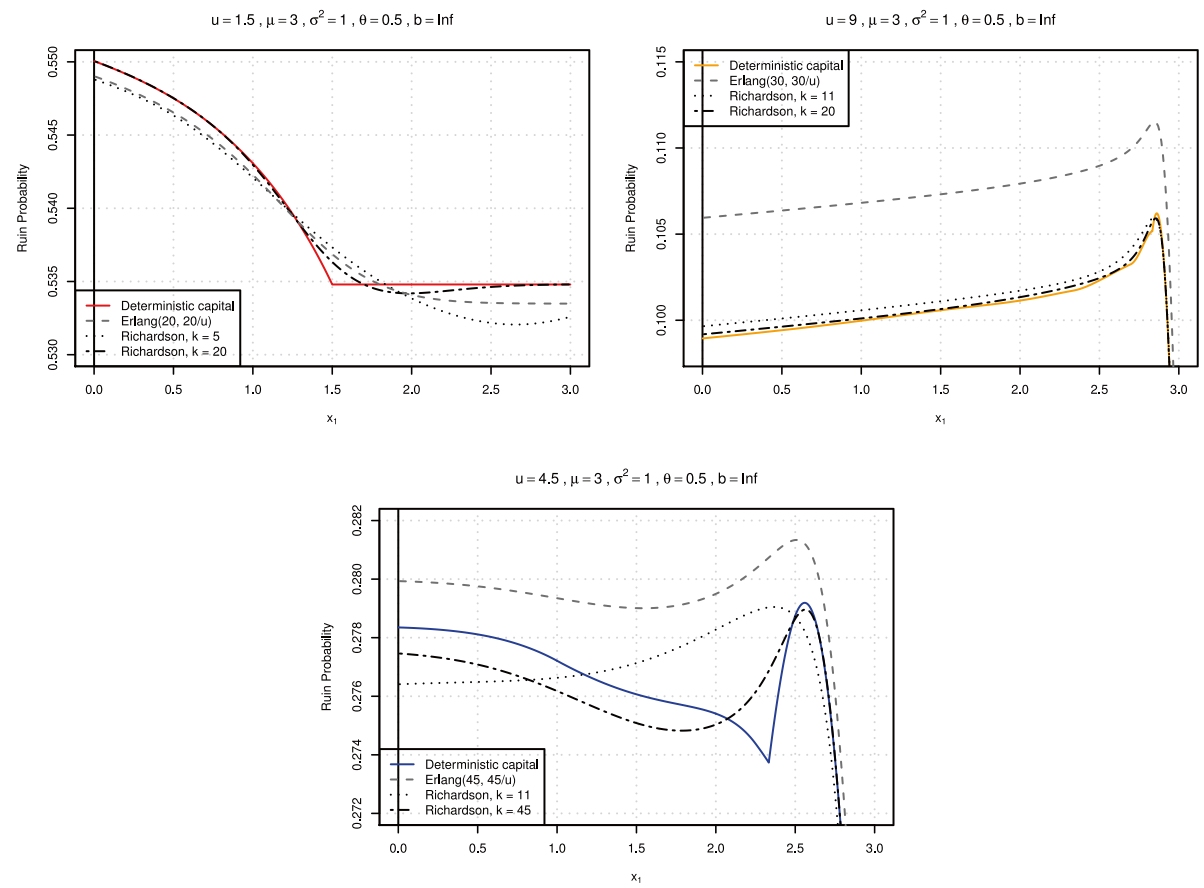

Fig. 5 Ruin probabilities as a function of $x_{1}$ for $\mu=3, \sigma^{2}=1, \theta=0.5$ and three levels of initial surplus $u$ (expected surplus $\mathbb{E}(U)=1 / s$, respectively)

$$
\begin{aligned}
\psi_{E}(k, s) & =\int_{0}^{\infty} \psi(u) \frac{s^{k}}{(k-1) !} u^{k-1} e^{-s u} d u=\int_{0}^{\infty} \psi(u) \frac{s^{k}}{(k-1) !}\left((-1)^{k-1} \frac{\partial^{k-1}}{\partial s^{k-1}} e^{-s u}\right) d u \\
& =\frac{s(-s)^{k-1}}{(k-1) !} \frac{\partial^{k-1}}{\partial s^{k-1}} \int_{0}^{\infty} \psi(u) e^{-s u} d u \\
& =\frac{s(-s)^{k-1}}{(k-1) !} \frac{\partial^{k-1}}{\partial s^{k-1}} \sum_{j=0}^{\infty} \frac{(-s)^{j}}{j !} \int_{0}^{\infty} u^{j} \mathbb{P}(L>u) d u \\
& =\frac{s(-s)^{k-1}}{(k-1) !} \frac{\partial^{k-1}}{\partial s^{k-1}} \sum_{j=0}^{\infty} \frac{(-s)^{j}}{(j+1) !} \mathbb{E}\left(L^{j+1}\right) \\
& =\sum_{j \geq k-1}(-1)^{j+k-1} \frac{s^{j+1}}{(j+1) !}\left(\begin{array}{c}
j \\
k-1
\end{array}\right) \mathbb{E}\left(L^{j+1}\right) .
\end{aligned}
$$

Therefore, one might try to understand the behavior of the $\psi_{E}(k, s)$ by analyzing the first, say, two terms of the previous series to obtain the approximation

$$
\psi_{E}(k, s) \approx \frac{s^{k}}{k !} \mathbb{E}\left(L^{k}\right)-\frac{s^{k+1}}{(k+1) !} k \mathbb{E}\left(L^{k+1}\right) .
$$

In the exponential case $k=1$, as the second moment of $l$ involves the first three moments of $X$, this means that for given $\mu_{1}, \mu_{2}$ one could infer about the behavior of $\psi_{U}(s)$ by simply analyzing the first non-given moment, i.e. $\mu_{3}$. We see that the same line of reasoning 
applied to the Erlang $(k)$ case needs the $(k+2)$-th moment of $X$, already for the above first two terms. This is unfortunate, as the deterministic $\psi(u)$ will only be obtained for $k \rightarrow \infty$, and we see that even in this simple approximation higher-order moments of $X$ already play a crucial role. This is in particular the case for moderate values of $\mathbb{E}(U)$, and in those cases we have indeed seen in the graphs above that a good approximation of the deterministic case needed large values of $k$.

Remark 4.2 When the goal is to approximate a deterministic initial surplus level, combinations of exponentials (i.e. densities of the form (9) but with $q_{i} \in \mathbb{R}, \sum_{i=1}^{n} q_{i}=1$ ) could a priori also be candidates for $U$, as that class is dense in the class of all distributions on the positive halfline, see e.g. Dufresne (2007)). Unfortunately, apart from the fact that an enormous number $n$ will be needed for a reasonable approximation of a deterministic $u$, the differing signs of $q_{i}$ in (10) then also do not allow to identify extremal distributions as in Section 3 .

\section{Conclusion}

In this paper we showed how randomization can be used to provide a solution to the Schmitter problem in ruin theory and its extension to higher moments. Linking this problem with established results in the theory of $m$-convex stochastic orders, we provided sharp bounds for the ruin probability under the assumption of an exponential initial surplus. For the more general case of Erlang distributed initial surplus, such analytical sharp bounds are not within reach. However, we showed how the deterministic classical case can be approximated by the simple expressions of the randomized case using Erlangization.

Acknowledgements The authors thank Hans Schmitter for several inspiring discussions on the topic. Financial support by the Swiss National Science Foundation Project 200021_191984 is gratefully acknowledged.

Funding Open access funding provided by University of Lausanne.

Open Access This article is licensed under a Creative Commons Attribution 4.0 International License, which permits use, sharing, adaptation, distribution and reproduction in any medium or format, as long as you give appropriate credit to the original author(s) and the source, provide a link to the Creative Commons licence, and indicate if changes were made. The images or other third party material in this article are included in the article's Creative Commons licence, unless indicated otherwise in a credit line to the material. If material is not included in the article's Creative Commons licence and your intended use is not permitted by statutory regulation or exceeds the permitted use, you will need to obtain permission directly from the copyright holder. To view a copy of this licence, visit http://creativecommons.org/licenses/by/4.0/.

\section{References}

Albrecher H, Beirlant J, Teugels J (2017) Reinsurance: Actuarial and Statistical Aspects. Wiley Series in Probability and Statistics. John Wiley \& Sons

Albrecher H, Cheung ECK, Thonhauser S (2013) Randomized observation periods for the compound Poisson risk model: the discounted penalty function. Scand Actuar J 2013(6):424-452

Albrecher H, Goffard PO (2021) On the profitability of selfish blockchain mining under consideration of ruin. Oper Res.

Albrecher H, Ivanovs J (2017) Strikingly simple identities relating exit problems for Lévy processes under continuous and Poisson observations. Stochastic Processes and their Applications 127(2):643-656

Asmussen S, Albrecher H (2009) Ruin Probabilities. Advanced series on statistical science \& applied probability, 14. World scientific, 2nd edition 
Asmussen S, Avram F, Usabel M (2002) Erlangian approximations for finite-horizon ruin probabilities. ASTIN Bulletin 32(2):267-281

Asmussen S, Steffensen M (2020) Risk and Insurance: A Graduate Text. Springer

Bowers NL (1969) An upper bound on the stop-loss net premium-actuarial note. Transactions of the Society of Actuaries, 21

Brockett PL, Cox SH (1984) Optimal ruin calculations using partial stochastic information. Transactions of the Society of Actuaries

Brockett PL, Cox SH (1985) Insurance calculations using incomplete information. Scand Actuar J 2:94-108

Brockett PL, Goovaerts MJ, Taylor GC (1991) The Schmitter problem. Astin Bulletin 21(1):129-132

Bühlmann H, Gagliardi B, Gerber HU, Straub E (1977) Some inequalities for stop-loss premiums. ASTIN Bulletin 9(1-2):75-83

Cai J, Garrido J (1998) Aging properties and bounds for ruin probabilities and stop-loss premiums. Insurance Math Econom 23(1):33-43

Carr P (1998) Randomization and the American put. Rev Fin Stud 11(3):597-626

De Vylder E (1982) Best upper bounds for integrals with respect to measures allowed to vary under conical and integral constraints. Insurance Math Econom 1(2):109-130

De Vylder E (1983) Maximization, under equality constraints, of a functional of a probability distribution. Insurance Math Econom 2(1):1-16

De Vylder E (1996) Advanced risk theory: A self-contained introduction. Editions de l'Université de Bruxelles

De Vylder E, Goovaerts M, Marceau E (1997) The bi-atomic uniform minimal solution of Schmitter's problem. Insurance Math Econ 20(1):59-78

De Vylder E, Goovaerts M, Marceau E (1997) The solution of Schmitter's simple problem: Numerical illustration. Insurance Math Econ 20(1):43-58

De Vylder E, Goovaerts MJ (1982) Analytical best upper bounds on stop-loss premiums. Insurance Math Econ 1(3):163-175

De Vylder E, Marceau E (1996) The numerical solution of Schmitter problems: Theory. Insurance Math Econ 19(1):1-18

Denuit M (2001) Laplace transform ordering of actuarial quantities. Insurance Math Econom 29(1):83-102

Denuit M, De Vylder E, Lefèvre C (1999) Extremal generators and extremal distributions for the continuous s-convex stochastic orderings. Insurance Math Econom 24(3):201-217

Denuit M, Lefèvre C, Shaked M (1998) The s-convex orders among real random variables, with applications. Math Ineq Their Appl 1(4):585-613

Denuit M, Lefèvre C, Shaked M (2000) On s-convex approximations. Adv Appl Probab 32(4):994-1010

Dufresne D (2007) Fitting combinations of exponentials to probability distributions. Appl Stoch Model Bus Ind 23(1):23-48

Eckberg AE (1977) Sharp bounds on laplace-stieltjes transforms, with applications to various queueing problems. Math Oper Res 2(2):135-142

Hürlimann W (1998) Extremal moment methods and stochastic orders. Application in Actuarial Science, Monograph manuscript

Ivanovs J (2013) A note on killing with applications in risk theory. Insurance Math Econom 52(1):29-34

Kaas R (1991) The Schmitter problem and a related problem: A partial solution. Astin Bulletin 21(1):133-146

Kaas R, Goovaerts MJ (1986) Best bounds for positive distributions with fixed moments. Insurance Math Econom 5(1):87-92

Kaas R, Goovaerts MJ, Dhaene J, Denuit M (2008) Modern actuarial risk theory: using R. Springer Science \& Business Media

Kaas R, Goovaerts MJ (1986) Bounds on stop-loss premiums for compound distributions. ASTIN Bulletin 16(1):13-17

Kaas R, Van Heerwaarden AE, Goovaerts M (1994) Ordering of actuarial risks. CAIRE, Brussels

Kaas R, Vanneste M, Goovaerts MJ (1992) Maximizing compound Poisson stop-loss premiums numerically with given mean and variance. ASTIN Bulletin 22(2):225-233

Kyprianou AE, Pistorius MR (2003) Perpetual options and Canadization through fluctuation theory. Ann Appl Probab 13(3):1077-1098

Lefèvre C, Hallin M, Narayan P (1986) On fractional linear bounds for probability generating functions. J Appl Probab 23:904-913

Lefèvre C, Loisel S, Montesinos P (2020) Bounding basis risk using s-convex orders on Beta-unimodal distributions. Preprint. HAL Id: hal-02611208 
Mnatsakanov RM (2008) Hausdorff moment problem: reconstruction of distributions. Stats Prob Lett 78(12):1612-1618

Müller A, Stoyan D (2002) Comparison methods for stochastic models and risks. Wiley Series in Probability and Statistics

Press WH, Teukolsky SA, Vetterling WT, Flannery BP (2007) Numerical recipes: The art of scientific computing, Third Edition. Cambridge University Press

Rolski T, Schmidli H, Schmidt V, Teugels JL (2009) Stochastic processes for insurance and finance. John Wiley \& Sons

Schmitter H (1990) The ruin probability of a discrete claims distribution with a finite number of steps. In XX1l ASTIN Colloquium, Montreux, Switzerland

Shaked M, Shanthikumar JG (1994) Stochastic orders and their applications. Academic Press, New York

Shaked M, Shanthikumar JG (2007) Stochastic orders. Springer Science \& Business Media

Stanford DA, Avram F, Badescu AL, Breuer L, Soares Da Silva A, Latouche G (2005) Phase-type approximations to finite-time ruin probabilities in the Sparre-Andersen and stationary renewal risk models. ASTIN Bulletin 35(1):131-144

Steenackers A, Goovaerts MJ (1991) Bounds on stop-loss premiums and ruin probabilities. Insurance Math Econom 10(2):153-159

Rolski T, Stoyan D (1976) On the comparison of waiting times in GI/G/1 queues. Oper Res 24(1):197-200

Whitt W (1983) Untold horrors of the waiting room: What the equilibrium distribution will never tell about the queue-length process. Manage Sci 29(4):395-408

Yarovaya E, Stoyanov JM, Kostyashin KK (2020) On conditions for a probability distribution to be uniquely determined by its moments. Theo Prob Its Appl 64(4):579-594

Publisher's Note Springer Nature remains neutral with regard to jurisdictional claims in published maps and institutional affiliations. 